\title{
Extension of a spatial evolutionary coordination game with neutral options
}

\author{
György Szabó ${ }^{1}$ and Balázs Király ${ }^{2}$ \\ ${ }^{1}$ Institute of Technical Physics and Materials Science, Centre for Energy Research, Hungarian Academy of Sciences, \\ P.O. Box 49, H-1525 Budapest, Hungary \\ ${ }^{2}$ Budapest University of Technology and Economics, Budafoki út 8, H-1111 Budapest, Hungary
}

(Received 5 February 2016; published 3 May 2016)

\begin{abstract}
The multiagent evolutionary games on a lattice are equivalent to a kinetic Ising model if the uniform pair interactions are defined by a two-strategy coordination game and the logit rule controls the strategy updates. Now we extend this model by allowing the players to use additional neutral strategies that provide zero payoffs for both players if one of them selects one of the neutral strategies. In the resulting $n$-strategy evolutionary games the analytical methods and numerical simulations indicate continuous order-disorder phase transitions when increasing the noise level if $n$ does not exceed a threshold value. For larger $n$ the system exhibits a first order phase transition at a critical noise level decreasing asymptotically as $2 / \ln (n)$.
\end{abstract}

DOI: 10.1103/PhysRevE.93.052108

\section{INTRODUCTION}

In the traditional coordination game [1-5] two players have two options to choose from, independently of each other. In the simplest case they get 1 (unit of payoff) if both choose the same option and lose 1 otherwise. In the multi-agent spatial evolutionary games [6-9] the players are located on the sites of a lattice, they play games with their neighbors, and are allowed to choose another strategy following a dynamical rule. It was recognized previously by many authors [10-15] that these models become equivalent to Ising-type models $[16,17]$ for a certain set of dynamical rules.

The above models can be extended by introducing additional strategies. In these systems the uniform interactions between the equivalent players are characterized by an $n \times n$ payoff matrix A [18]. One of the well-investigated extensions is represented by the family of Potts models [19], where A is an $n \times n$ unit matrix. For the voluntary version of these games [20,21], we introduce a third option corresponding to declining to play games. In this case, the original $2 \times 2$ payoff matrix is extended by a third row and column consisting of zeros.

The decomposition of the payoff matrix into a linear combination of orthogonal matrices (see Refs. [22-27]), representing basis games, has thrown light on the importance of matrices describing coordination-type interactions between the $i$ th and $j$ th strategies of the two players. All these matrices, as well as their linear combinations, are symmetric $\left(A_{i j}=A_{j i}\right)$. Consequently, these matrices define potential games [28-31] for which we can apply the tools of statistical physics for the investigation of the macroscopic behavior. More precisely, these systems evolve into the Boltzmann distribution $[28,32]$ if the dynamics of strategy distribution is controlled by a logit rule.

In this paper, our attention will be focused on the general features of the above-mentioned set of $n \times n$ basis games possessing a coordination-type interaction between the first and second strategies, whereas the rest of the strategies provide zero income for both players. It will be shown that these evolutionary games on a square lattice exhibit Ising-type order-disorder phase transitions if the number of strategies is less than a threshold value. For larger $n$, however, we can observe a first-order transition at a critical noise level going to zero in the limit $n \rightarrow \infty$. The formalism and the general features of these models are described in Sec. II. In Sec. III we briefly survey the methods used in the present work. The results of the different approaches are compared in Sec. III D, and in Sec. IV we summarize the main conclusions and raise some questions.

\section{THE MODELS}

We study spatial evolutionary games with equivalent players located at the sites $x$ of a square lattice with $N=L \times L$ points in the limit $N \rightarrow \infty$. Each player $x$ has $n$ pure strategies that are denoted by the standard $n$-dimensional unit vectors as

$$
\mathbf{s}_{x}=\mathbf{s}_{x, 1}=\left(\begin{array}{c}
1 \\
0 \\
\vdots \\
0
\end{array}\right), \cdots, \mathbf{s}_{x, n}=\left(\begin{array}{c}
0 \\
0 \\
\vdots \\
1
\end{array}\right) .
$$

Using one of their strategies the players play games with their four nearest neighbors, and the total income of player $x$ is given as

$$
\tilde{u}_{x}\left(\mathbf{s}_{x}\right)=\sum_{\delta} \mathbf{s}_{x} \cdot \mathbf{A} \mathbf{s}_{x+\delta},
$$

where the summation runs over the nearest neighbors located at the sites $x+\delta$ and the payoffs are defined by the following $n \times n$ matrix:

$$
\mathbf{A}=\left(\begin{array}{rrrrr}
1 & -1 & 0 & \cdots & 0 \\
-1 & 1 & 0 & \cdots & 0 \\
0 & 0 & 0 & \cdots & 0 \\
\vdots & \vdots & \vdots & \ddots & \vdots \\
0 & 0 & 0 & \cdots & 0
\end{array}\right)
$$

In words, the components $A_{i j}$ are zero except for four ones, namely $A_{11}=A_{22}=-A_{12}=-A_{21}=1$. For $n=2$ the present payoff matrix represents a two-strategy coordination game when the rational players are forced to choose the same strategy. For $n>2$, if one of the players chooses her $i$ th strategy $(2<i \leqslant n)$, then both players receive zero income. Henceforth, the latter strategies are called neutral strategies. 
Similar games can be introduced when deriving a payoff matrix from $\mathbf{A}$ by exchanging its $i$ th row and column with the first (or second) one simultaneously. All these games have similar features. Namely, the payoff matrices are symmetric $\left(A_{i j}=A_{j i}\right)$ and there exists coordination-type interactions for a suitable $2 \times 2$ subgame when both players are constrained to use the same strategy pair providing +1 or -1 income for them. Evidently, in the latter cases the rest of the strategies are neutral.

Several linear combinations of these basis games have been investigated previously on a square lattice for $n=3$ and 4 [24-26]. Now we study evolutionary games with pair interactions [Eq. (3)] for larger $n$.

In the present multiagent system the potential of the whole system is given as

$$
U(\mathbf{s})=\frac{1}{2} \sum_{x, \delta} \mathbf{s}_{x} \cdot \mathbf{A} \mathbf{s}_{x+\delta},
$$

for any strategy profile $\mathbf{s}=\left\{\mathbf{s}_{x}\right\}$ defining the individual strategy $\mathbf{s}_{x}$ for each player, because the two-player potential matrix is equivalent to $\mathbf{A}$. This potential summarizes the individual incentives for the unilateral strategy changes. More precisely, if player $x$ modifies her strategy from $\mathbf{s}_{x}$ to $\mathbf{s}_{x}^{\prime}$, then

$$
\tilde{u}_{x}\left(\mathbf{s}_{x}\right)-\tilde{u}_{x}\left(\mathbf{s}_{x}^{\prime}\right)=U(\mathbf{s})-U\left(\mathbf{s}^{\prime}\right),
$$

where $\mathbf{s}_{y}=\mathbf{s}_{y}^{\prime}$ for all $y \neq x$ in the strategy profiles $\mathbf{s}$ and $\mathbf{s}^{\prime}$. The random sequential strategy updates for the players are defined by the logit rule, when the randomly selected player $x$ modifies her strategy to $\mathbf{s}_{x}^{\prime}$ with a probability

$$
w\left(\mathbf{s}_{x}^{\prime}\right)=\frac{e^{\tilde{u}_{x}\left(\mathbf{s}_{x}^{\prime}\right) / K}}{\sum_{\mathbf{s}_{x}} e^{\tilde{u}_{x}\left(\mathbf{s}_{x}\right) / K}},
$$

favoring exponentially her higher individual income with assuming fixed strategies in the neighborhood [33-37]. The value of $K$ quantifies the strength of noises. The application of this evolutionary rule drives the system onto a stationary state where the probability $p(\mathbf{s})$ of the strategy profile $\mathbf{s}$ is determined by the Boltzmann distribution $[28,32]$, that is,

$$
p(\mathbf{s})=\frac{e^{U(\mathbf{s}) / K}}{\sum_{\mathbf{s}^{\prime}} e^{U\left(\mathbf{s}^{\prime}\right) / K}} .
$$

On the analogy of the ferromagnetic Ising model the present system has two equivalent ordered preferred Nash equilibria (ground states) when $U(\mathbf{s})$ reaches its largest value. In these states all the players choose the first $\left(\mathbf{s}_{x}=\mathbf{s}_{x, 1}\right)$ or second $\left(\mathbf{s}_{x}=\mathbf{s}_{x, 2}\right)$ strategy in the limit $K \rightarrow 0$ for any finite $n$. If $K$ is increased then these systems exhibit an Ising-type order-disorder phase transition at $K_{c}(n)$ for $n=2$, 3 , and $4[16,24,26,38]$. These critical transitions are generally quantified by evaluating the frequencies $\rho_{i}$ for all the strategies. In the mentioned cases, $\left|\rho_{1}-\rho_{2}\right|$ vanishes algebraically if $K$ approaches $K_{c}(n)$ from below. More precisely, $\left|\rho_{1}-\rho_{2}\right| \propto$ $\left(K_{c}(n)-K\right)^{\beta}$ (with $\beta=1 / 8$ ) in the close vicinity of the critical points.

For $K>K_{c}(n) \rho_{1}=\rho_{2}>\rho_{i}(2<i \leqslant n)$ when all the neutral strategies are present with the same frequency. Evidently, all the strategy frequencies become equivalent $\left(\rho_{j}=1 / n\right.$ for $1 \leqslant j \leqslant n$ ) in the limit $K \rightarrow \infty$ when the strategy selections are not influenced by the payoffs.

\section{METHODS}

\section{A. Mean-field and pair approximations}

The cluster variation methods (for details see Refs. [27,3941]) give a general framework for both the mean-field and pair approximations. In these approximative methods the homogeneous lattice system is described by one-site probabilities $p_{1}(i)=\rho_{i}$ of finding strategy $i$ at any sites (on the translation invariant lattice) and by two-site configuration probabilities $p_{2}(i, j)$ of finding strategy pair $(i, j)$ on two neighboring sites. Due to the translation and rotation symmetries, the condition of normalization,

$$
\sum_{i} p_{1}(i)=1,
$$

and compatibility conditions,

$$
p_{1}(i)=\sum_{j} p_{2}(i, j)=\sum_{j} p_{2}(j, i)
$$

the number of independent parameters can be reduced drastically. For example, in the present systems the one-site configuration probabilities can be expressed by two independent parameters $\left(\rho_{2}\right.$, and $\rho_{3}$ for $\left.n>2\right)$ as

$$
\begin{aligned}
& p_{1}(1)=1-\rho_{2}-(n-2) \rho_{3}, \\
& p_{1}(2)=\rho_{2}, \\
& p_{1}(i)=\rho_{3}, \quad \text { if } i>2 .
\end{aligned}
$$

Similarly, for the pair approximation we have five independent two-site configuration probabilities to evaluate.

In the spirit of cluster variation methods the values of the independent configuration probabilities can be determined by finding the extremum of a thermodynamic potential,

$$
\Phi=\mathcal{U}+K \mathcal{S},
$$

where $\mathcal{U}$ and $\mathcal{S}$ are the average value of the potential $\left(\mathcal{U}=\sum_{\mathbf{s}} p(\mathbf{s}) U(\mathbf{s})\right)$ and the entropy $\left(\mathcal{S}=-\sum_{\mathbf{s}} p(\mathbf{s}) \ln p(\mathbf{s})\right)$, respectively. In the one-site approximation $\Phi$ can be approximated by

$$
\Phi^{(1)}=2 N \sum_{i, j} p_{1}(i) p_{1}(j) A_{i j}-N K \sum_{i} p_{1}(i) \ln p_{1}(i) .
$$

The equilibrium values of $\rho_{2}$ and $\rho_{3}$ can be obtained by finding the maximum of $\Phi^{(1)}$ with respect to these quantities. In general, the equations

$$
\frac{\partial \Phi^{(1)}}{\partial \rho_{2}}=0 \text { and } \frac{\partial \Phi^{(1)}}{\partial \rho_{3}}=0
$$

have several solutions. In the latter cases we should select the solutions providing the highest values of $\Phi^{(1)}$.

The above method can be straightforwardly extended for the two-site approximation when the corresponding thermodynamic potential is given as

$$
\begin{aligned}
\Phi^{(2)}= & 2 N \sum_{i, j} p_{2}(i, j) A_{i j}-2 N K \sum_{i, j} p_{2}(i, j) \ln p_{2}(i, j) \\
& +3 N K \sum_{i} p_{1}(i) \ln p_{1}(i),
\end{aligned}
$$




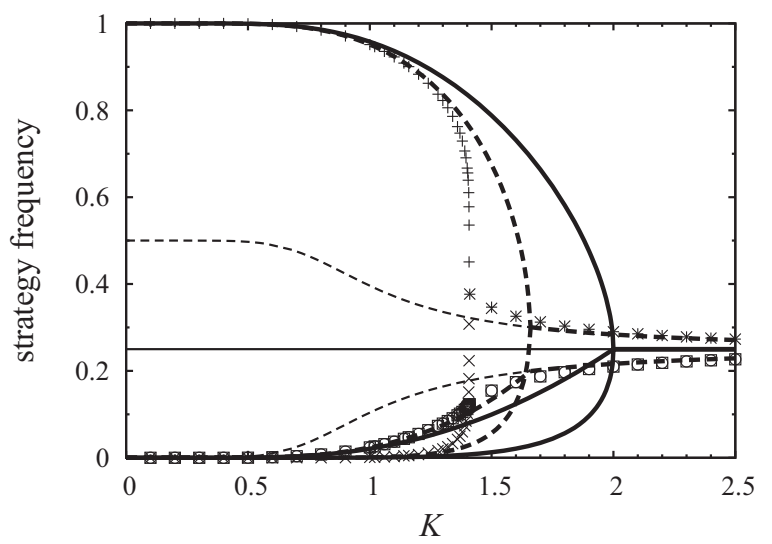

FIG. 1. Strategy frequencies vs. $K$ show a continuous orderdisorder phase transition for $n=4$. The symbols indicate MC data, the predictions of the one- and two-site approximations are illustrated by solid and dashed lines. Thick (thin) lines represent stable (unstable) solutions.

in the limit $N \rightarrow \infty$. The equilibrium values of the independent two-site configuration probabilities $p_{2}(i, j)$ can be evaluated in the same way as drafted above.

The results of numerical solutions are compared with the MC data (discussed later) in Figs. 1 and 2 for $n=4$ [26] and 6. In both cases the approximations reproduce the MC data qualitatively well. Namely, these systems undergo a continuous order-disorder phase transition when $K$ is increased.

For $n=4$ the three methods predict the following critical noise levels: $K_{c}^{(1 \mathrm{~s})}(4)=2, K_{c}^{(2 \mathrm{~s})}(4)=1.65(2)$, and $K_{c}^{(\mathrm{MC})}(4)=$ $1.4076(2)$. For $n=6$ the same results are: $K_{c}^{(1 \mathrm{~s})}(6)=$ $4 / 3, K_{c}^{(2 \mathrm{~s})}(6)=1.25(2)$, and $K_{c}^{(\mathrm{MC})}(6)=1.102(2)$. The $K$ dependence of the strategy frequencies refers to continuous order-disorder phase transition as shown in Fig. 2.

These results illustrate some general properties that remain valid for larger $n$. For example, if $\rho_{1} \rightarrow 1$ in the low noise

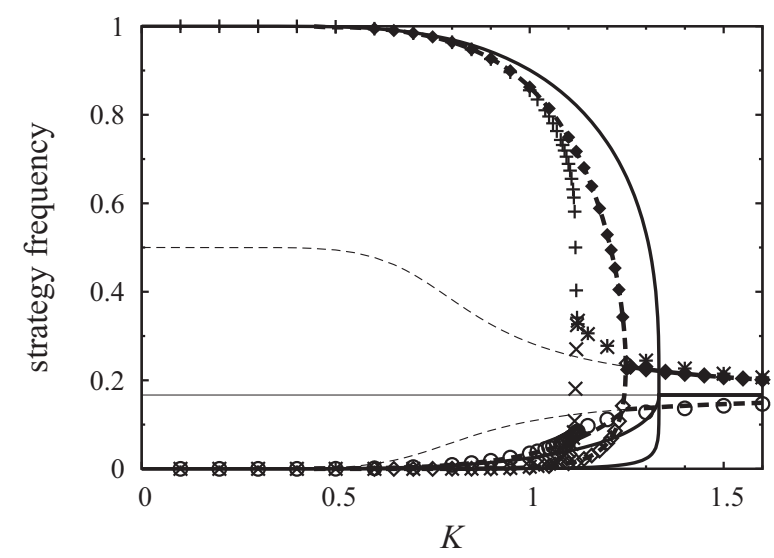

FIG. 2. Strategy frequencies as a function of $K$ for $n=6$. Notations are the same as described in the caption of Fig. 1. Notice the agreement between the predictions of pair approximation and Monte Carlo data (closed and empty diamonds denote the frequencies of the first and second strategies) obtained for $N=160000$ sites on a random regular graph.

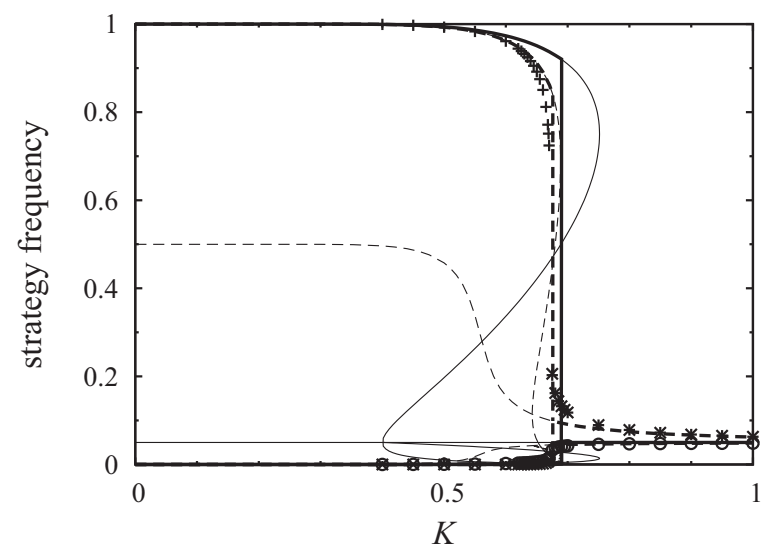

FIG. 3. Variations in strategy frequencies for $n=20$. Notations are the same as described in the caption of Fig. 1.

limit then $\rho_{2}<\rho_{i}=\rho_{3}$ for $2<i \leqslant n$ in agreement with the expectation. In the disordered phase $\left(K>K_{c}(n)\right)$, however, $\rho_{1}=\rho_{2}>\rho_{i}=\rho_{3}$. Furthermore, $K_{c}(n)$ decreases with $n$ for all three approaches.

In light of the previous results (for references see Ref. [27]), the pair approximation can exactly describe the $K$-dependence of strategy frequencies on sufficiently large random regular graphs. This expectation is confirmed by Monte Carlo data in Fig. 2.

The above numerical calculations have also been performed for several larger $n$ values when the one- and two-site approximations predict a first-order phase transition as it is illustrated in Fig. 3 if $n=20$. Here the thin lines show all the possible solutions of Eqs. (13) while the thick lines denote the preferred ones where the $\Phi^{(1)}$ (or $\Phi^{(2)}$ ) reaches the maximum value. The vertical thick lines refer to step-like changes in the strategy frequencies. Notice that here the MC data indicate a continuous phase transition. The numerical solutions of the mean-field approximation indicate that the continuous phase transition becomes a first-order one for $n>6$. At the same time the pair approximations predict a first-order transition if $n>10$. On the contrary, the MC simulations indicate Ising-type critical transitions for both threshold values of $n$ as discussed later.

We have to emphasize that the mean-field approximations predict an uncorrelated random strategy distribution $\left(\rho_{i}=\right.$ $1 / n)$ for any values of $n$. The latter feature is related to the fact that the average value of the potential remains zero $(\mathcal{U}=0)$ in these states. Considering the Taylor series expansion at these solutions, we can deduce the following analytical formula for the critical points:

$$
K_{c}^{(1 \mathrm{~s})}(n)=\frac{8}{n},
$$

in agreement with the numerical values given above for $n=4$ and 6 , when this approach predicts a mean-field-type continuous phase transition. Notice, furthermore, that quadratic deviations in $\rho_{1}$ and $\rho_{2}$ are predicted by the mean-field approximation for the unstable solutions at $K_{c}^{(1 \mathrm{~s})}(n)$ defined by Eq. (15).

The above results also imply the possibility of deriving an asymptotic estimation of $K_{c}^{(1 \mathrm{~s})}(n)$ for large values of $n$. 
Namely, we may assume that the ordered phase (with $p_{1}(1)=$ 1 and $p_{1}(i)=0$ for $i>1$ when $\mathcal{U} / N=2$ and $\mathcal{S} / N=0$ ) transforms into an uncorrelated disordered state $\left(p_{1}(i)=\right.$ $1 / n \forall i$ with $\mathcal{U}^{(1)} / N=0$ and $\mathcal{S}^{(1)} / N=\ln n$ ) at a noise level $K$ where the contribution of entropy to $\Phi^{(1)}$ exceeds the average value of the potential in the ordered phase. This approach predicts a first-order phase transition at

$$
K_{c}^{(1 \mathrm{~s})}(n) \simeq \frac{2}{\ln n},
$$

if $n \gg 1$. Notice that the high entropy stabilizes the disordered phase at a low noise level for sufficiently large $n$. A similar mechanism is utilized for high entropy alloys [42-44] considered a promising family of materials for several technical purposes.

\section{B. Monte Carlo simulations}

Monte Carlo (MC) simulations are performed on a square lattice of $N=L \times L$ sites with periodic boundary conditions. The linear size of the system is varied from $L=300$ to 1000. During the simulations we have determined the strategy frequencies $\left(\rho_{i}\right)$ by averaging over a sampling time $t_{s}$ after a relaxation time $t_{r}$. The values of $t_{s}$ and $t_{r}$ are varied from $10^{4}$ to $10^{5}$ Monte Carlo steps (MCS) where in $1 \mathrm{MCS}$ each player receives a chance to modify her strategy once on average. The larger system sizes and the longer run times are used in the close vicinity of the critical phase transitions in order to suppress the undesired effects of the diverging fluctuations, correlation lengths, and relaxation time. Additionally, we have used different initial states to achieve adequate results characterizing the system behavior in the final stationary state in the limit $N \rightarrow \infty$.

We have performed a series of MC simulations varying the noise level $K$ and the number of strategies. The numerical results are illustrated in Fig. 4 for four different values of $n$. Notice that, contrary to the results of mean field and pair approximations, the MC data indicate a continuous phase transition at $n=20$. Additionally, one can observe a fast

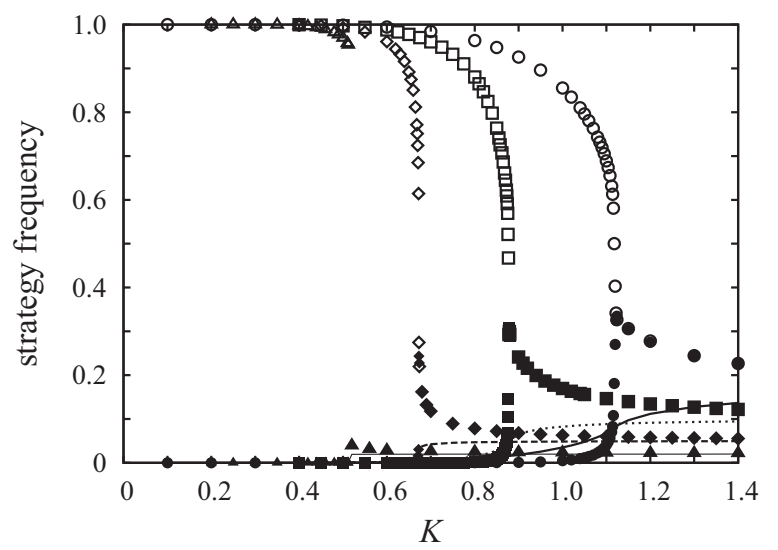

FIG. 4. Monte Carlo results for the strategy frequencies as a function of $K$ for $n=6$ (circles), 10 (boxes), 20 (diamonds), and 50 (triangles). Open (closed) symbols indicate the frequencies of strategy 1 [Eq. (2)]. The frequencies of the neutral strategies are denoted by thick solid, dotted, dashed, and thin lines.

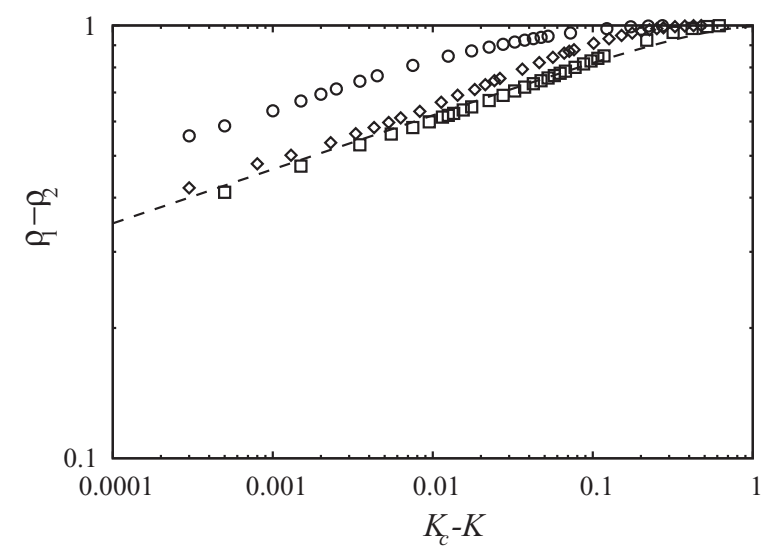

FIG. 5. Log-log plots of the order parameters $\left|\rho_{1}-\rho_{2}\right|$ versus $K_{c}(n)-K$. The MC results are illustrated by boxes, diamonds, and circles for $n=6,10$, and 20, respectively. The dashed line illustrates the exact (Onsager [38]) result for $n=2$.

decrease of $\rho_{1}=\rho_{2}$ in the disordered phase in the close vicinity of $K_{c}(n)$ for both $n=10$ and 20 .

The Ising-type behaviors $\left[\left|\rho_{1}-\rho_{2}\right| \propto\left(K_{c}(n)-K\right)^{1 / 8}\right.$ $[16,45]$ in the limit $\left.\left(K_{c}(n)-K\right) \rightarrow+0\right]$ are demonstrated by a log-log plot in Fig. 5 for several values of $n$.

The numerical results refer to a critical phase transition that belongs to the Ising universality class in agreement with the results obtained previously for $n=3$ and 4 [24-26].

In order to give a suitable estimate for the threshold value of $n$ we have performed further systematic numerical investigations. In the close vicinity of the phase transitions we used larger system sizes $(L \geqslant 1400)$ and longer run times $\left(t_{r}=t_{s}>2 \times 10^{5} \mathrm{MCS}\right)$ in the simulations. According to the Monte Carlo data (see Fig. 6) one can conclude that the continuous phase transition becomes a first-order one if $n>n_{\text {th }}=27$. We emphasize, however, that we need further time-consuming numerical investigations if we wish to clarify the details of how the Ising-type critical transition turns into a first-order transition. In agreement with our expectations the presence of a first-order phase transition is accompanied with

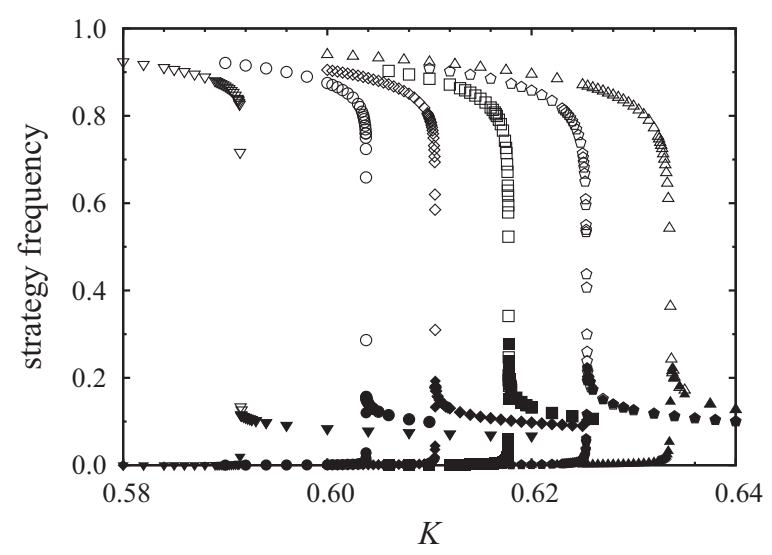

FIG. 6. Monte Carlo results of frequencies for the first and second strategies are illustrated by empty and closed symbol pairs for $n=30$, $28,27,26,25$, and 24 (from left to right). 

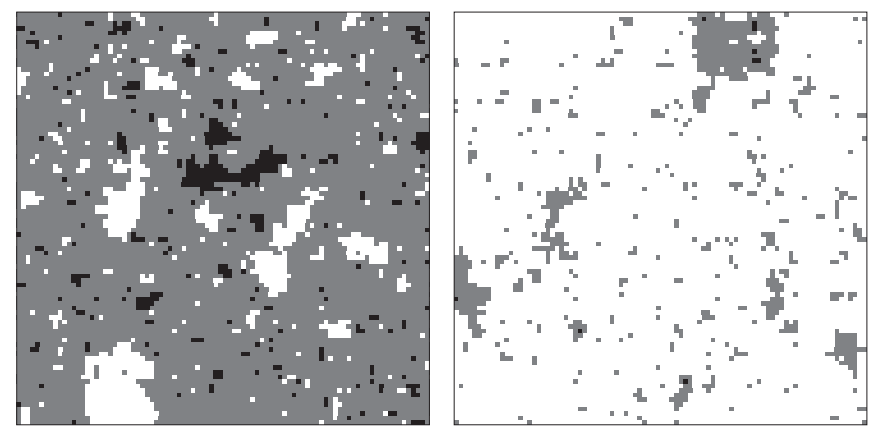

FIG. 7. These snapshots illustrate how the metastable (disordered) states evolve toward one of the stable (ordered) phases throughout a nucleation process on a square lattice for $n=50$. For both pictures the white and black boxes refer to strategy 1 and 2 , whereas all the neutral strategies $(2<i \leqslant n)$ are denoted by a uniform gray color. On the left snapshot the system is started from a random strategy distribution at $K=0.5<K_{c}(50)$ and after the formation of islands with the preferred ordered strategy arrangements the system evolves into a pattern composed dominantly of black and white domains with growing sizes. For the right-hand snapshot the simulation is started from a homogeneous ordered (white) phase at a noise level $K=0.52>K_{c}(50)$ and here one can observe several (gray) domains of the disordered phase that grow at a constant radial velocity if their sizes are sufficiently large.

the appearance of hysteresis phenomena when increasing or decreasing $K$ through the critical points.

Previous systematic investigations have already clarified the dynamics of the ordering processes especially for the Ising and Potts models [17,19,46-50]. The nucleation theorems [51-53] are developed to describe the formation of domains of the thermodynamically preferred phase that are sufficiently large to grow with a constant average radial velocity. In the present models the relevance of these phenomena are illustrated in Fig. 7. The snapshots show the strategy distributions at the times when the sufficiently large domains of the emerging phases are well recognizable.

The left-hand snapshot demonstrates the appearance of two types (black and white) of domains in the sea of the neutral strategies in the nucleation period of the evolutionary process when the expansion of the preferred domains is sustained by the suitable motion of steps along the horizontal and vertical interfaces. The right-hand snapshot of Fig. 7 illustrates an opposite process when the neutral phase expands to the disadvantage of the ordered strategy arrangement(s). Both processes are preceded by the formation of sufficiently large islands of the thermodynamically preferred phases.

In agreement with the theoretical predictions [51-53] the appearance of sufficiently large domains requires longer time in the vicinity of $K_{c}(n)$ for larger $n$ and this phenomenon causes technical difficulties in the determination of $K_{c}(n)$. These troubles can be avoided by starting the simulations from an artificial initial state containing large domains of all the possible final states. In those cases we can quantify the invasion velocity between the competing phases as it is used for the determination of phase diagrams in many other evolutionary games [37,54-61].

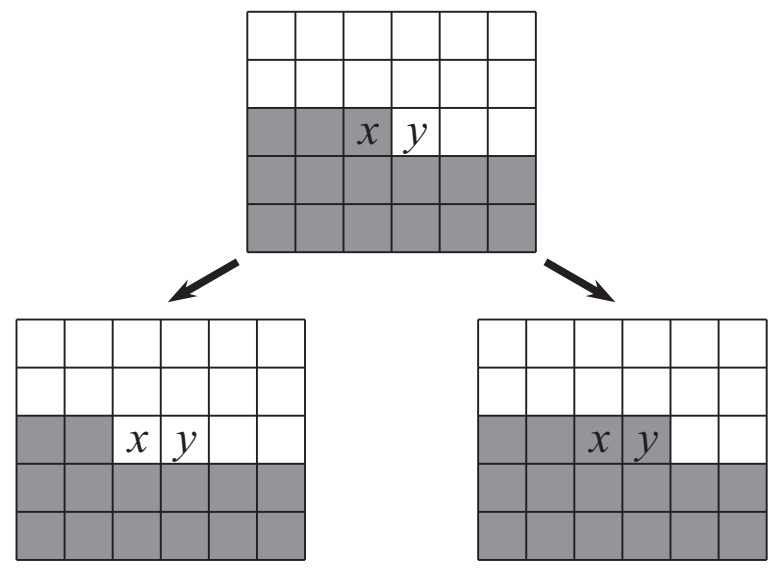

FIG. 8. Two competing elementary processes along a step-like interface separating the homogeneous ordered (white) phase and the disordered phase composed of neutral (gray) strategies.

\section{Average invasion velocity}

The visualization of the microscopic variation in patterns plotted in Fig. 7 indicates clearly that the most relevant processes occur at the steps located along the horizontal and vertical interfaces. Figure 8 illustrates a horizontal interface with one step that separates the (white) ordered $\left(\mathbf{s}_{y^{\prime}}=\mathbf{s}_{y, 1}\right)$ and (gray) disordered ( $\mathbf{s}_{x^{\prime}}=\mathbf{s}_{x, j}$ with $2<j \leqslant n$ ) territories. Along this step the pair interactions favor the transition $\mathbf{s}_{x} \rightarrow \mathbf{s}_{x, 1}$ that occurs with a probability

$$
w\left(\mathbf{s}_{x} \rightarrow \mathbf{s}_{x, 1}\right)=\frac{e^{2 / K}}{\sum_{\mathbf{s}_{x}} e^{u_{x}\left(\mathbf{s}_{x}\right)}},
$$

at a noise level $K$ for the logit rule, Eq. (6). On the other hand, player $y$ will choose one of the neutral strategies from the $(n-2)$ options with a probability

$$
w\left(\mathbf{s}_{y} \rightarrow \mathbf{s}_{x, j}\right)=\frac{n-2}{\sum_{\mathbf{s}_{y}} e^{u_{y}\left(\mathbf{s}_{y}\right)}} .
$$

Notice that the denominators of Eqs. (17) and (18) are equivalent as both players $x$ and $y$ have two neighbors each belonging to the competing phases and at low noises we can neglect the roles of the transitions $\mathbf{s}_{x} \rightarrow \mathbf{s}_{x, 2}$ and/or $\mathbf{s}_{y} \rightarrow \mathbf{s}_{x, 2}$. At a low noise level $\left(K<K_{c}^{\text {(if) }}(n)\right)$ the ordered phase will expand on average if $w\left(\mathbf{s}_{x} \rightarrow \mathbf{s}_{x, 1}\right)>(n-2) w\left(\mathbf{s}_{y} \rightarrow \mathbf{s}_{x, j}\right)$. In the opposite case the disordered phase will conquer the ordered phase. However, there is a balance between these processes at the critical point $K_{c}^{(\mathrm{if})}(n)$ that can be estimated as

$$
K_{c}^{(\text {if) }}(n) \simeq \frac{2}{\ln (n-2)},
$$

for large values of $n$. This asymptotic behavior is similar to those we obtained from the mean field calculations and the small difference is related to the neglected correlations occurring in both the ordered and the disordered phases.

\section{Comparison of asymptotic behaviors}

In order to check the asymptotic values of $K_{c}(n)$ we have run additional MC simulations for $n=100$ and 500 predicting first order transitions at $K_{c}(100)=0.435(3)$ and 


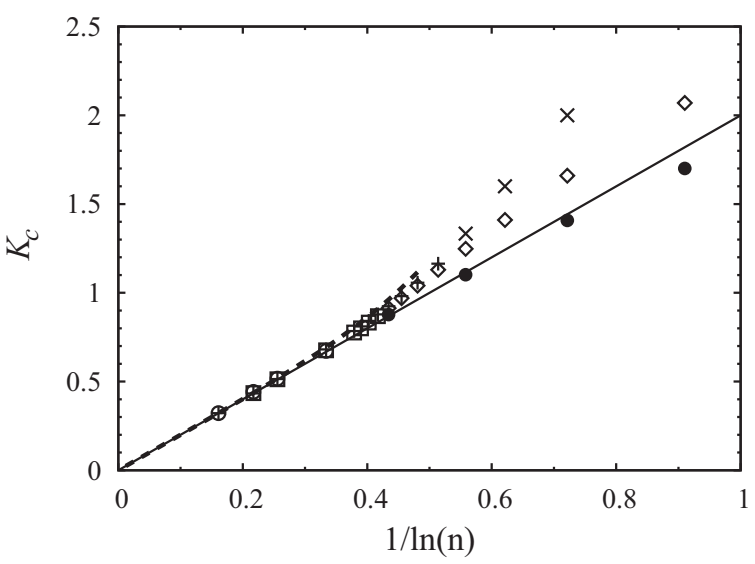

FIG. 9. Critical points versus $1 / \ln (n)$ for $n>3$. Open and closed circles denote MC results for the first- and second-order phase transitions. Pluses and crosses as well as open boxes and diamonds show the corresponding predictions of the mean-field and pair approximations. Solid and dashed lines illustrate the predictions of Eqs. (16) and (19).

$K_{c}(500)=0.322(2)$. Figure 9 compares the results we have deduced by using the approaches discussed above. The most striking message of this plot is that the mean-field results can be used as a rule of thumb for the estimation of the critical transition points for $n>3$.

\section{SUMMARY}

Using different methods we have studied the order-disorder phase transitions in a special set of evolutionary potential games on a square lattice when the dynamics is controlled by a logit rule. The linear combinations of similar pair interactions span the class of coordination-type interactions in symmetric $n \times n$ games [26,27]. The latter set of games are orthogonal to the other three classes of symmetric matrix games characterized by self- or cross-dependent payoffs, or by cyclic dominance among the strategies. The present games are located along $n(n-1) / 2$ lines (within the subset of coordination type interactions) in the $n^{2}$-dimensional space of payoff parameters. All these systems have a coordinationtype interaction between a strategy pair while the remaining strategies provide zero payoffs for the interacting players. When varying the noise level $K$ these games exhibit universal properties dependent on $n$. Due to the mentioned symmetry our analysis is restricted to the models where the coordination-type interaction exists only between the first and second strategies while the other $(n-2)$ strategies are neutral.

For $n=2$ the present system is identical to the Ising model that undergoes a critical phase transition representing the Ising universality class [45]. For larger $n$ the same power-law behavior is observed in the $K$-dependence of $\left|\rho_{1}-\rho_{2}\right|$ when approaching the critical point $K_{c}(n)$ if $n$ does not exceed a threshold value $n_{\text {th }}$ that could be estimated by the mean-field and pair approximations. The MC simulations, however, have indicated a higher threshold value, namely $n_{\text {th }}=27$. For $n>n_{\text {th }}$ a first-order phase transition occurs at $K_{c}(n)$ decreasing asymptotically as $K_{c}(n) \simeq 2 / \ln (n)$ in the limit $n \rightarrow \infty$. The mean-field calculations have clearly indicated that this asymptotic behavior is related to the logarithmic divergence of entropy $(\mathcal{S} / N \simeq \ln n)$ in the disordered phase.

Finally, we emphasize that within the class of coordination interactions two typical behaviors can be distinguished [26]. In the first type of cases, the single maximum value of $V_{i j}$ is located in the main diagonal of the payoff matrix (e.g., $\left.\max \left(V_{i j}\right)=V_{11}\right)$ when the corresponding strategy forms a homogeneous phase at low noises that varies smoothly toward the disordered phase if $K$ is increased. In the second type of cases, $\max \left(V_{i j}\right)=V_{p q}=V_{q p}(p \neq q)$ when the strategy pair $(p, q)$ can form two equivalent sublattice-ordered strategy arrangements (at low noises on the square lattice) reminiscent of an antiferromagnetic spin system. A similar strategy distribution can occur on other lattices (or bipartite graphs), which can be divided into two equivalent sublattices. These systems can be transformed into a ferromagnetic system if the labels $p$ and $q$ are exchanged in one of the sublattices. The latter systems possess an Ising-type critical phase transition due to the mentioned symmetry relation.

In addition to the present set of coordination-type games one can distinguish other curious games that are analogous to the $Q$-state Potts models. In the latter cases, however, one can introduce additional neutral strategies, too. It is conjectured that most of the above conclusions can be adapted when analyzing the latter models.

\section{ACKNOWLEDGMENTS}

This work was supported by the Hungarian National Research Fund (OTKA Grant No. TK-101490).
[1] R. Gibbons, Game Theory for Applied Economists (Princeton University Press, Princeton, NJ, 1992).

[2] M. J. Osborn and A. Rubinstein, A Course in Game Theory (MIT Press, Cambridge, MA, 1994).

[3] J. W. Weibull, Evolutionary Game Theory (MIT Press, Cambridge, MA, 1995).

[4] R. Cooper, Coordination games (Cambridge University Press, Cambridge, 1998).

[5] K. Sigmund, The Calculus of Selfishness (Princeton University Press, Princeton, NJ, 2010).
[6] M. A. Nowak, Evolutionary Dynamics (Harvard University Press, Cambridge, MA, 2006).

[7] G. Szabó and G. Fáth, Phys. Rep. 446, 97 (2007).

[8] C. P. Roca, J. A. Cuesta, and A. Sánchez, Phys. Life Rev. 6, 208 (2009).

[9] B. Allen and M. A. Nowak, EMS Surv. Math. Sci. 1, 113 (2014).

[10] S. Galam, Y. Gefen, and Y. Shapir, Math. J. Sociol. 9, 1 (1982).

[11] A. V. M. Herz, J. Theor. Biol. 169, 65 (1994).

[12] W. A. Brock and S. N. Durlauf, Rev. Econ. Stud. 68, 235 (2001).

[13] G. Weisbuch and D. Stauffer, Physica A 384, 542 (2007). 
[14] S. Galam and B. Walliser, Physica A 389, 481 (2010).

[15] S. Grauwin, D. Hunt, E. Bertin, and P. Jensen, Adv. Complex Syst. 14, 529 (2011).

[16] C. Domb, in Phase Transitions and Critical Phenomena, Vol. 3, edited by C. Domb and M. S. Green (Academic Press, London, 1974), pp. 357-484.

[17] R. J. Glauber, J. Math. Phys. 4, 294 (1963).

[18] J. von Neumann and O. Morgenstern, Theory of Games and Economic Behaviour (Princeton University Press, Princeton, 1944).

[19] F. Y. Wu, Rev. Mod. Phys. 54, 235 (1982).

[20] C. Hauert, S. De Monte, J. Hofbauer, and K. Sigmund, Science 296, 1129 (2002).

[21] G. Szabó and C. Hauert, Phys. Rev. E 66, 062903 (2002).

[22] O. Candogan, I. Menache, A. Ozdaglar, and P. A. Parrilo, Math. Oper. Res. 36, 474 (2011).

[23] S.-H. Hwang and L. Rey-Bellet, arXiv:1106.3552v2.

[24] G. Szabó, K. S. Bodó, B. Allen, and M. A. Nowak, Phys. Rev. E 90, 042811 (2014).

[25] J. Vukov, L. Varga, B. Allen, M. A. Nowak, and G. Szabó, Phys. Rev. E 92, 012813 (2015).

[26] G. Szabó, K. S. Bodó, B. Allen, and M. A. Nowak, Phys. Rev. E 92, 022820 (2015).

[27] G. Szabó and I. Borsos, Phys. Rep. 624, 1 (2016).

[28] L. E. Blume, Games Econ. Behav. 5, 387 (1993).

[29] L. E. Blume, Games Econ. Behav. 11, 111 (1995).

[30] D. Monderer and L. S. Shapley, Games Econ. Behav. 14, 124 (1996).

[31] D. Cheng, Automatica 50, 1793 (2014).

[32] L. E. Blume, Games Econ. Behav. 44, 251 (2003).

[33] G. W. Brown, in Activity Analysis of Production and Allocation, edited by T. C. Koopmans (Wiley, New York, 1951), pp. 373376.

[34] D. Monderer and L. S. Shapley, J. Econ. Theory 68, 258 (1996).

[35] M. Sysi-Aho, J. Saramäki, J. Kertész, and K. Kaski, Eur. Phys. J. B 44, 129 (2005).

[36] W. H. Sandholm, Games 1, 3 (2010).

[37] G. Szabó and A. Szolnoki, J. Theor. Biol. 299, 81 (2012).

[38] L. Onsager, Phys. Rev. 65, 117 (1944).

[39] R. Kikuchi and S. Brush, J. Chem. Phys. 47, 195 (1967).
[40] T. Morita, Prog. Theor. Phys. Suppl. 115, 27 (1994).

[41] D. de Fontaine, in Theory and Applications of the Cluster Variation and Path Probability Methods, edited by J. L. MorinLopez and J. M. Sanchez (Springer, New York, 1996), p. 125.

[42] R. Carroll, C. Li, C.-W. Tsai, J. Yeh, J. Antonaglis, B. A. W. Brinkman, M. LeBlanc, X. Xie, S. Chen, P. K. Liaw et al., Sci. Rep. 5, 16997 (2015).

[43] O. N. Senkov, G. B. Wilks, D. B. Miracle, C. P. Chuang, and P. K. Liaw, Intermetallics 18, 1758 (2010).

[44] J.-W. Yeh, JOM 65, 1759 (2013).

[45] H. E. Stanley, Introduction to Phase Transitions and Critical Phenomena (Clarendon Press, Oxford, 1971).

[46] I. M. Lifshitz, J. Expt. Theor. Phys. (USSR) 42, 1354 (1962) [Sov. Phys. JETP 15, 939 (1962)].

[47] P. C. Hohenberg and B. I. Halperin, Rev. Mod. Phys. 49, 435 (1977).

[48] S. E. Allen and J. W. Cahn, Acta Metall. 27, 1085 (1979).

[49] J. D. Gunton and M. S. Miguel, in Phase Transitions and Critical Phenomena, Vol. 8, edited by C. Domb and J. L. Lebowitz (Academic Press, New York, 1983).

[50] A. J. Bray, Adv. Phys. 43, 357 (1994).

[51] K. Binder and H. Müller-Krumbhaar, Phys. Rev. B 9, 2328 (1974).

[52] F. F. Abraham, Homogeneous Nucleation Theory (Academic Press, New York, 1974).

[53] P. A. Rikvold, H. Tomita, S. Miyashita, and S. W. Sides, Phys. Rev. E 49, 5080 (1994).

[54] H. Matsuda, N. Ogita, A. Sasaki, and K. Sato, Progr. Theor. Phys. 88, 1035 (1992).

[55] S. P. Ellner, A. Sasaki, Y. Haraguchi, and H. Matsuda, J. Math. Biol. 36, 469 (1998).

[56] G. Szabó and G. A. Sznaider, Phys. Rev. E 69, 031911 (2004).

[57] F. Fu, M. A. Nowak, and C. Hauert, J. Theor. Biol. 266, 358 (2010).

[58] W.-X. Wang, X. Ni, Y.-C. Lai, and C. Grebogi, Phys. Rev. E 83, 011917 (2011).

[59] G. Szabó, A. Szolnoki, and L. Czakó, J. Theor. Biol. 317, 126 (2013).

[60] A. Dobrinevski, M. Alava, T. Reichenbach, and E. Frey, Phys. Rev. E 89, 012721 (2014).

[61] A. Szolnoki and M. Perc, Proc. R. Soc. B 282, 20151975 (2015). 